\title{
Hybrid Projective Synchronization of Fractional-Order Chaotic Systems with Time Delay
}

\author{
Li-xin Yang ${ }^{1,2}$ and Jun Jiang ${ }^{1}$ \\ ${ }^{1}$ State Key Laboratory for Strength and Vibration, Xian Jiaotong University, Xi'an 710049, China \\ ${ }^{2}$ School of Mathematic and Statistics, Tianshui Normal University, Tianshui 741001, China \\ Correspondence should be addressed to Li-xin Yang; jiaodayanglixin@163.com
}

Received 21 December 2012; Revised 22 May 2013; Accepted 7 June 2013

Academic Editor: Jinde Cao

Copyright (C) 2013 L.-x. Yang and J. Jiang. This is an open access article distributed under the Creative Commons Attribution License, which permits unrestricted use, distribution, and reproduction in any medium, provided the original work is properly cited.

The hybrid projective synchronization for fractional-order chaotic systems with time delay is investigated in this paper. On the basis of stability analysis of fractional-order systems and pole placement technique, a novel and general approach is proposed. The hybrid projective synchronization of fractional-order chaotic and hyperchaotic systems with time delay is achieved via designing an appropriate controller. Corresponding numerical results are presented to demonstrate the effectiveness of the proposed synchronization scheme. Furthermore, the influence of the fractional order on the synchronization process is discussed. The result reveals that the fractional order has a significant effect on the synchronization speed.

\section{Introduction}

Fractional calculus is a topic being more than 300 years old. The idea of fractional calculus has been known since the regular calculus, with the first reference probably being associated with Leibniz and L'Hospital in 1965 where halforder derivative was mentioned. Compared with the integer order model, fractional-order derivatives provide a wonderful instrument for the description of memory and hereditary properties of many processes. Fractional-order systems are shown to widely exist in various fields of the real world and have attracted increasing interests in recent years [14]. For instance, viscoelastic system [5], models of real capacitor [6], real inductor [7], and so forth. As a matter of fact, many fractional-order differential systems behave chaotically, such as the fractional-order Liu system [8], the fractional-order Chen system [9], and the fractional-order Chua system [10] [11-17]. Also, there has been a great interest in the synchronization of chaotic dynamical systems due to their wide range of applications, such as signal processing and automatic control engineering [18, 19]. Even though the synchronization of the integer-order chaotic systems has been investigated by many researchers. In [20], the authors investigated projective synchronization of a class of delayed chaotic systems via impulsive control. After that, He et al. [21] studied the impulsive synchronization of two nonidentical chaotic systems with time-varying delay; they derived the sufficient conditions for impulsive synchronization with a bound on the synchronization error and so forth $[22,23]$.

As for the synchronization of fractional-order chaotic systems, we notice that there are already some literature discussing this topic; however, it has not received adequate attention. In [24], the authors studied the phase synchronization in coupled Sprott chaotic systems presented by fractional differential equations; after that, Zhang et al. [25] pointed out the complete synchronization problem of fractional-order system; they have found a kind of interesting nonlinear phenomenon-hybrid synchronization in linearly coupled fractional-order chaotic systems. Recently, Wang et al. [26] studied the hybrid projective synchronization of fractional-order chaotic system. They realized that the slave system can be synchronized with the projection of the master system generated through state transformation. Due to the complexity of the different scale factors, this type of synchronization can be used to extend binary digital to M-nary digital communication for getting fast communication. So, it is necessary to discuss 
the hybrid projective synchronization of fractional-order chaotic systems.

On the other hand, it is known that time delay should be and must be an important factor that affects the synchronization capability of chaotic system in the real world. Therefore, in most cases, it is more reasonable to require the slave system to synchronize the master system with a time delay. However, little research effort has been done about the hybrid projective synchronization of the fractional-order chaotic (hyperchaotic) systems with time delay. Chen et al. [27] discussed the lag synchronization of structurally nonequivalent chaotic systems with time delays in detail. In [28], chaos synchronization of uncertain fractional-order chaotic systems with time delay based on adaptive fuzzy sliding mode control is investigated.

Inspired by the aforementioned works, we investigate the hybrid projective synchronization of fractional-order chaotic systems with time delay in this paper. Based on the stability theory of fractional-order systems, we propose an appropriate controller to realize the hybrid projective synchronization of fractional-order chaotic systems with time delay. In addition, we find that the fractional order of chaotic systems would influence the synchronization process. The bigger the fractional order is, the better the synchronization performance turns out. Also, the synchronization rate of integer-order chaotic (hyperchaotic) system is much faster than that of the fractional-order one, and the synchronization effect is much better than that of the fractional-order ones.

The paper is organized as follows. In Section 2, a scheme for hybrid projective synchronization of chaotic systems with time delay is proposed. In Section 3, numerical simulations are given to illustrate the effectiveness of the approach. The paper is concluded in Section 4.

\section{Scheme Design for Hybrid Projective Synchronization}

There exist many definitions for fractional derivatives. The Riemann-Liouville definition and the Caputo definition are the two most commonly used ones. In this paper, the Caputo definition is adopted for derivatives which is introduced briefly in the following [29]:

$$
D_{*}^{\alpha} f(t)=\frac{1}{\Gamma(n-\alpha)} \int_{t_{0}}^{t}(t-\tau)^{\alpha-n+1} f^{(n)}(\tau) d \tau,
$$

for $n-1 \leq \alpha<n$, where $\Gamma(\cdot)$ is the Gamma function.

Consider two general fractional-order chaotic systems which are referred to as the drive and response systems, respectively, in the form of

$$
\begin{gathered}
D_{*}^{q} \mathbf{x}(t)=\mathbf{A x}(t)+\mathbf{F}(\mathbf{x}(t)), \\
D_{*}^{q} \mathbf{y}(t)=\mathbf{B y}(t)+\mathbf{G}(\mathbf{y}(t))+\mathbf{U}(t),
\end{gathered}
$$

where $\mathbf{x}(t)=\left(x_{1}(t), x_{2}(t), \ldots, x_{n}(t)\right)^{T} \in \mathbf{R}^{n}, \mathbf{y}(t)=\left(y_{1}(t)\right.$, $\left.y_{2}(t), \ldots, y_{n}(t)\right)^{T} \in \mathbf{R}^{n}$ are the state vectors, $\mathbf{F}: \mathbf{R}^{n} \rightarrow \mathbf{R}^{n}$, $\mathbf{G}: \mathbf{R}^{n} \rightarrow \mathbf{R}^{n}$ are continuous vector functions, $\mathbf{A} \in \mathbf{R}^{n \times n}$,
$\mathbf{B} \in \mathbf{R}^{n \times n}$ are constant matrices, and $\mathbf{U}(t)=\left(u_{1}(t), u_{2}(t)\right.$, $\left.\ldots, u_{n}(t)\right)^{T} \in \mathbf{R}^{n}$ is a controller to be designed later.

Definition 1 (see [30]). For the given drive system (2) and response system (3), if there exist a real scaling matrix $\Lambda$ and a delay time $\tau, \lim _{t \rightarrow \infty}\|\mathbf{e}(t)\|=\|\mathbf{y}(t)-\Lambda \mathbf{x}(t-\tau)\|=0$. It is to say that the hybrid projective synchronization with time delay is achieved between system (2) and system (3).

Define the error vector as $\mathbf{e}(t)=\mathbf{y}(t)-\mathbf{\Lambda} \mathbf{x}(t-\tau)$; so, the error dynamical system is determined as

$$
\begin{aligned}
D_{*}^{q} \mathbf{e}(t)= & \mathbf{B e}(t)+\mathbf{B} \mathbf{\Lambda} \mathbf{x}(t-\tau)+\mathbf{G}(\mathbf{y}(t)) \\
& -\boldsymbol{\Lambda} \mathbf{A} \mathbf{x}(t-\tau)-\mathbf{\Lambda} \mathbf{F}(\mathbf{x}(t-\tau))+\mathbf{U}(t) .
\end{aligned}
$$

It is noticed that the hybrid projective synchronization between the two systems (2) and (3) can be translated into the analysis of the asymptotical stability of zero solution of the error system (4). In the next step, a suitable controller is designed to ensure the asymptotical stability to zero solution of the error system (4) based on the stability theorem of fractional-order systems.

Some remarks on Definition 1 are as follows.

Remark 2. If $\boldsymbol{\Lambda}=\mathbf{I}$ and $\boldsymbol{\Lambda}=-\mathbf{I}$ (I is identity matrix), hybrid projective synchronization will be reduced to the projective synchronization and projective antisynchronization, respectively.

Remark 3. If $\tau=0$, hybrid projective synchronization with time delay degenerates to the hybrid projective synchronization.

Remark 4. The scaling matrix $\Lambda$ can be arbitrarily chosen in this paper; thus, we select a simpler form which is the diagonal matrix.

Lemma 5 (see [29]). Consider a general autonomous system that can be described by

$$
D_{*}^{q} \mathbf{x}=\mathbf{A x},
$$

with $x(0)=x_{0}$, where $\mathbf{x} \in \mathbf{R}^{n}$ is the state variable, $\mathbf{A} \in \mathbf{R}^{n \times n}$ is constant matrix, and $q$ indicates the fractional order; one has the following.

Case 1. Asymptotically stable if and only if $\left|\arg \left(\lambda_{i}(\mathbf{A})\right)\right|>$ $q \pi / 2, i=1,2,3, \ldots, n$, where $\arg \left(\lambda_{i}(\mathbf{A})\right)$ denotes the argument of the eigenvalue $\lambda_{i}$ of $\mathbf{A}$. In case of $q=1$, the previous stability agrees with the well-known results for ordinary linear differential systems.

Case 2. Stable if and only if either it is asymptotically stable or those critical eigenvalues which satisfy $\left|\arg \left(\lambda_{i}(\mathbf{A})\right)\right|=q \pi / 2$, $i=1,2,3, \ldots$ and have geometric multiplicity one.

Theorem 6. For a given scaling matrix $\Lambda$ and time delay $\tau$, system (2) and system (3) can achieve hybrid projective 
synchronization with time delay for any initial values, by using the following controller:

$$
\begin{aligned}
\mathbf{U}= & \Lambda \mathbf{A x}(t-\tau)+\Lambda \mathbf{F x}(t-\tau) \\
& -\mathbf{B} \Lambda \mathbf{x}(t-\tau)-\mathbf{G}(\mathbf{y}(t))+\mathbf{K e}(t),
\end{aligned}
$$

where $\mathbf{K} \in \mathbf{R}^{n \times n}$ is a gain matrix.

Proof. Submitting controller (6) into system (4), the error dynamical system can be rewritten as

$$
\begin{aligned}
D_{*}^{q} \mathbf{e}(t)= & \mathbf{B} \mathbf{e}(t)+\mathbf{B} \Lambda \mathbf{x}(t-\tau)+\mathbf{G}(\mathbf{y}(t)) \\
& -\mathbf{A} \mathbf{A} \mathbf{x}(t-\tau)-\mathbf{\Lambda} \mathbf{F}(\mathbf{x}(t-\tau))+\mathbf{U}(t) \\
= & \mathbf{B} \mathbf{e}(t)+\mathbf{B} \mathbf{\Lambda} \mathbf{x}(t-\tau)+\mathbf{G}(\mathbf{y}(t))-\mathbf{\Lambda} \mathbf{A} \mathbf{x}(t-\tau) \\
& -\boldsymbol{\Lambda} \mathbf{F}(\mathbf{x}(t-\tau))+\Lambda \mathbf{A x}(t-\tau)+\Lambda \mathbf{F} \mathbf{x}(t-\tau) \\
& -\mathbf{B} \Lambda \mathbf{x}(t-\tau)-\mathbf{G}(\mathbf{y}(t))+\mathbf{K e}(t) \\
= & (\mathbf{B}+\mathbf{K}) e .
\end{aligned}
$$

Due to Lemma 5, if we choose an appropriate gain matrix $\mathbf{K}$, all the eigenvalues $\lambda_{i}$ of $\mathbf{B}+\mathbf{K}$ satisfy $\left|\arg \left(\lambda_{i}\right)\right|>q \pi / 2, i=$ $1,2,3, \ldots n$, that is $\lim _{t \rightarrow \infty}\|e\|=0$. It is to say that systems (2) and (3) realize the hybrid projective synchronization with time delay by the controller (6).

\section{Examples and Numerical Simulations}

In this section, numerical examples will be given to verify the effectiveness of hybrid projective synchronization with time delay in fractional-order chaotic systems. First of all, we intend to investigate the hybrid projective synchronization behavior between the fractional-order chaotic Chen and Lü systems; furthermore, we will study the hybrid projective synchronization between the fractional-order hyperchaotic Lorenz system and a new fractional-order system.

3.1. Hybrid Projective Synchronization between the FractionalOrder Chen System and the Fractional-Order Lü System. In 2004, Li and Peng and $\mathrm{Li}$ and Chen studied the chaotic behaviors of the chaotic Chen system with fractional order, which is described as follows:

$$
\begin{aligned}
& D_{*}^{q} x_{1}(t)=a\left(x_{2}-x_{1}\right), \\
& D_{*}^{q} x_{2}(t)=(c-a) x_{1}-x_{1} x_{3}+c x_{2}, \\
& D_{*}^{q} x_{3}(t)=x_{1} x_{2}-b x_{3},
\end{aligned}
$$

where $0<q<1$ is the fractional order and $x_{1}, x_{2}, x_{3}$ are state variables, when $a=35, b=3, c=28$, and $q=0.9$; system (8) exhibits chaotic behaviors as shown in Figure 1.

The fractional-order Lü system is given by

$$
\begin{aligned}
& D_{*}^{q} y_{1}(t)=d\left(y_{2}-y_{1}\right), \\
& D_{*}^{q} y_{2}(t)=-y_{1} y_{3}+h y_{2}, \\
& D_{*}^{q} y_{3}(t)=y_{1} y_{2}-f y_{3} .
\end{aligned}
$$

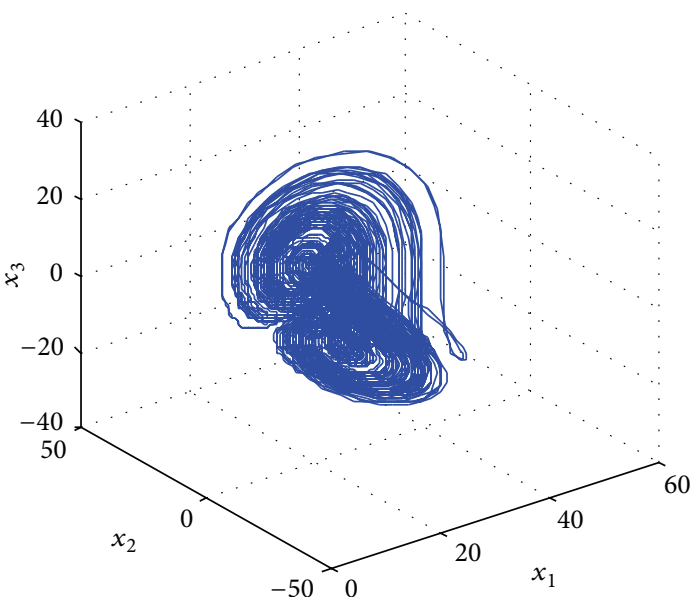

FIGURE 1: The attractor of the Chen system with $q=0.9$.

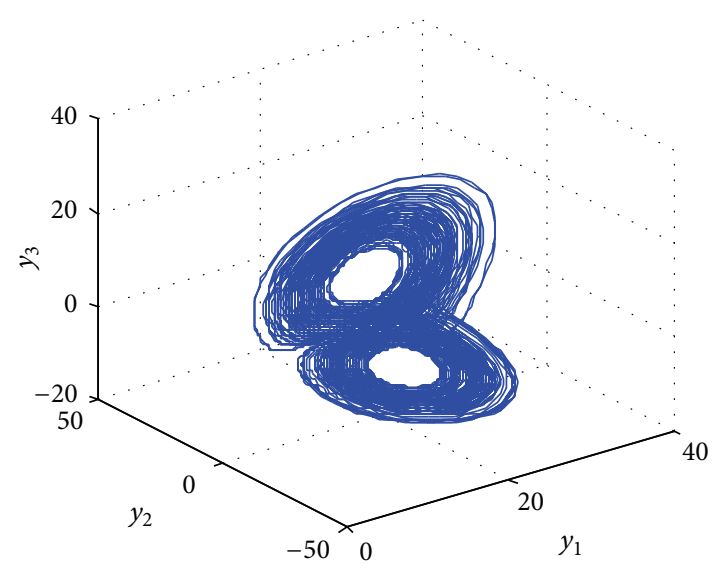

FIGURE 2: The attractor of the Lü system with $q=0.9$.

System (9) exhibits a chaotic attractor for $d=36, h=20$, $f=3$. Figure 2 displays the chaotic attractor of the fractionalorder Lü chaotic system with the order $q=0.9$.

We take that system (8) is the drive system and the response system is the fractional-order chaotic Lü system, which is given as follows:

$$
\begin{aligned}
& D_{*}^{q} y_{1}(t)=d\left(y_{2}-y_{1}\right)+u_{1}, \\
& D_{*}^{q} y_{2}(t)=-y_{1} y_{3}+h y_{2}+u_{2}, \\
& D_{*}^{q} y_{3}(t)=y_{1} y_{2}-f y_{3}+u_{3},
\end{aligned}
$$

where $u_{i}(i=1,2,3)$ are the control functions to be determined in the later discussion.

Compare systems (8) and (10) with systems (2) and (3), one can have

$$
\begin{array}{cl}
\mathbf{A}=\left[\begin{array}{ccc}
-a & a & 0 \\
c-a & c & 0 \\
0 & 0 & -b
\end{array}\right], & \mathbf{F}(\mathbf{x})=\left[\begin{array}{c}
0 \\
-x_{1} x_{3} \\
x_{1} x_{2}
\end{array}\right], \\
\mathbf{B}=\left[\begin{array}{ccc}
-d & d & 0 \\
0 & h & 0 \\
0 & 0 & -f
\end{array}\right], & \mathbf{G}(\mathbf{y})=\left(\begin{array}{c}
0 \\
-y_{1} y_{3} \\
y_{1} y_{2}
\end{array}\right) .
\end{array}
$$




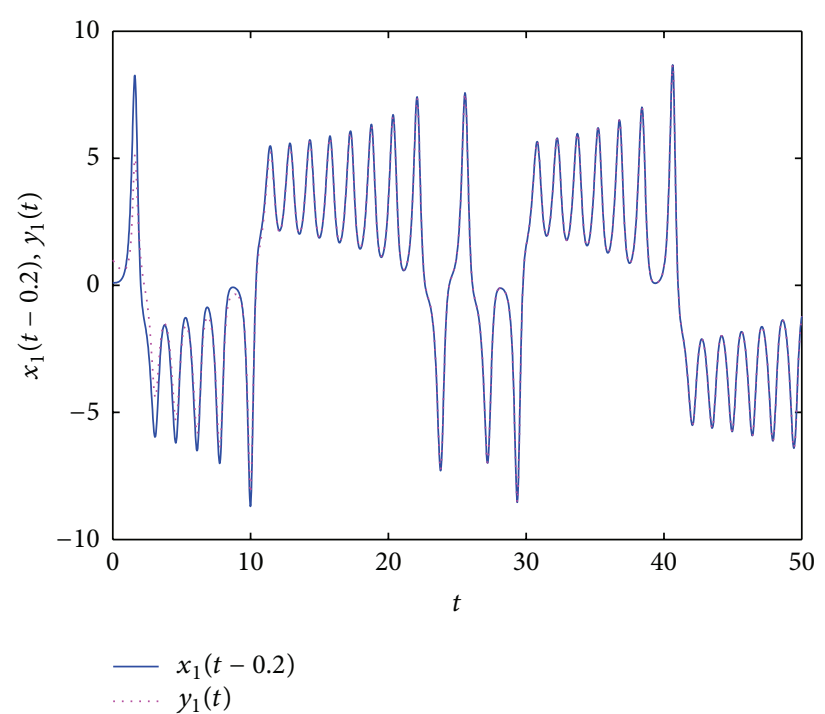

(a) State variables of $x_{1}$ and $y_{1}$

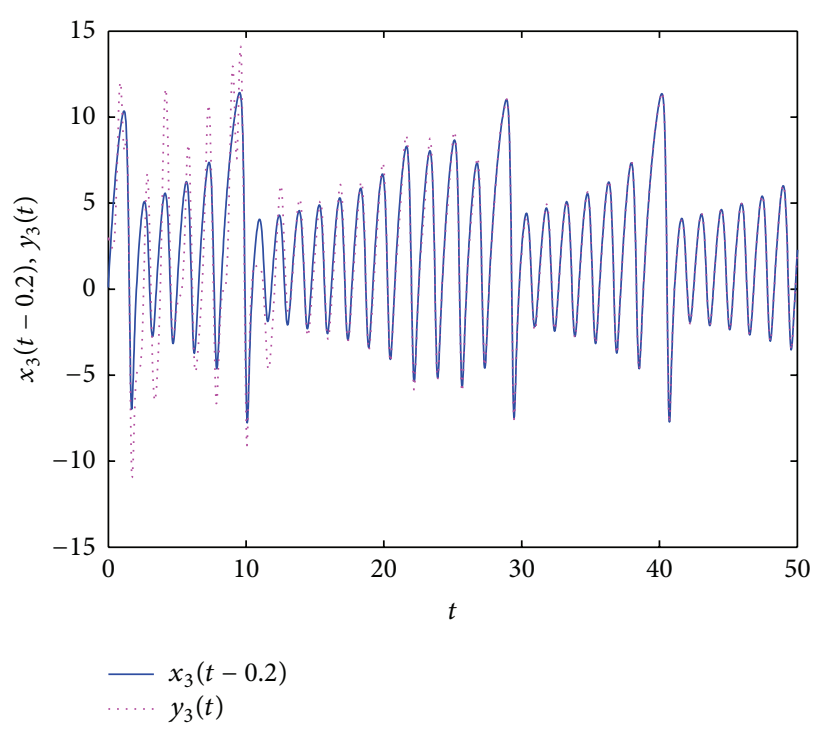

(c) State variables of $x_{3}$ and $y_{3}$

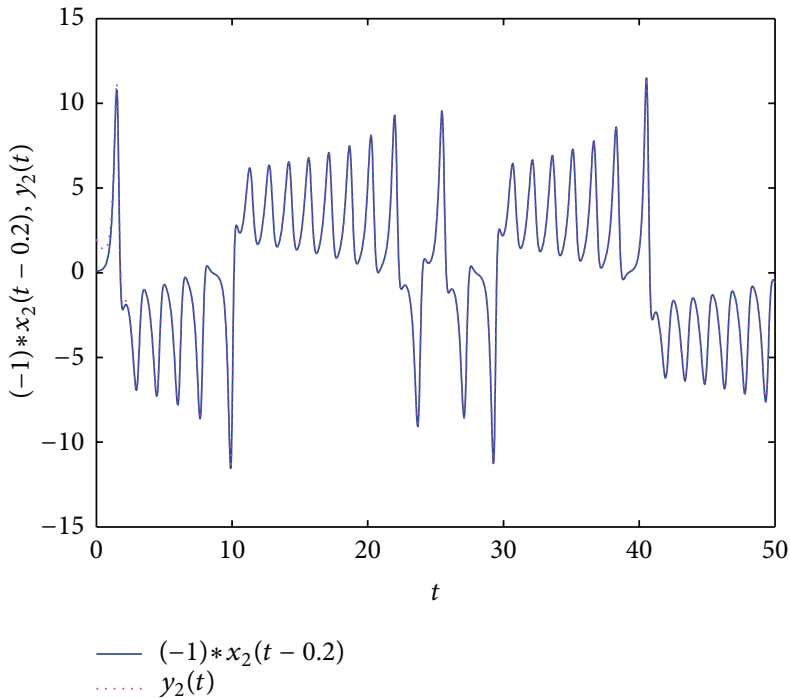

(b) State variables of $x_{2}$ and $y_{2}$

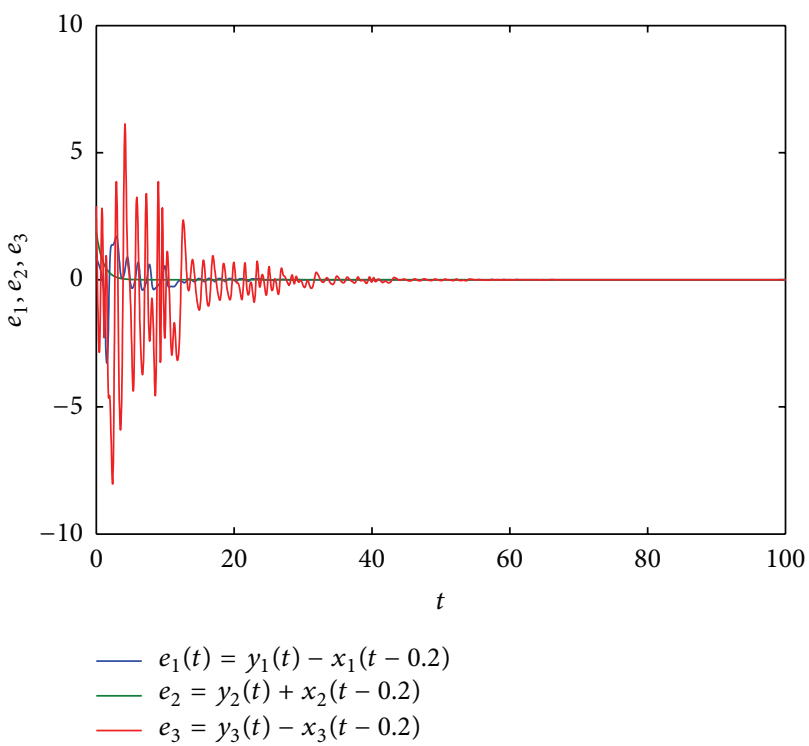

(d) Error states of the drive system (8) and response system (10)

FIGURE 3: The state synchronization variables and errors between the drive system (8) and response system (10) with time delay $\tau=0.2$ and scaling factors $\left(\lambda_{1}, \lambda_{2}, \lambda_{3}\right)=(1,-1,1)$.

We choose the following scaling matrix:

$$
\Lambda=\left[\begin{array}{ccc}
1 & 0 & 0 \\
0 & -1 & 0 \\
0 & 0 & 1
\end{array}\right] \text {. }
$$

Then, the error system $\mathbf{e}(\mathrm{t})=\mathbf{y}(\mathrm{t})-\mathbf{\Lambda} \mathbf{x}(t-\tau)$ can be obtain as

$$
\begin{aligned}
& e_{1}=y_{1}-\left(x_{1}(t-\tau)\right), \\
& e_{2}=y_{2}+\left(x_{2}(t-\tau)\right), \\
& e_{3}=y_{3}-\left(x_{3}(t-\tau)\right) .
\end{aligned}
$$

According to Theorem 6, the control function is considered as $\Upsilon=\Lambda \mathbf{A x}(t-\tau)+\mathbf{A} \mathbf{F} \mathbf{x}(t-\tau)-\mathbf{B} \mathbf{\Lambda} \mathbf{x}(t-\tau)+\mathbf{K e}(t), \mathbf{K} \in \mathbf{R}^{n \times n}$.
Hence, the error dynamical system is given in the following form:

$$
D_{*}^{q} \mathbf{e}=(\mathbf{B}+\mathbf{K}) \mathbf{e} .
$$

It can be seen that there exist a gain matrix $\mathbf{K}$ such that system (8) and system (10) realize the hybrid projective synchronization. For example, we can set all the eigenvalues $\lambda_{i}=-1$ of $\mathbf{B}+\mathbf{K}$ with the matrix

$$
\mathbf{K}=\left[\begin{array}{ccc}
35 & -35 & 0 \\
0 & -21 & 0 \\
0 & 0 & 2
\end{array}\right]
$$


Which satisfy $\left|\arg \left(\lambda_{i}\right)\right|>0.9 \pi / 2, i=1,2,3, \ldots$ In other words, we can realize the hybrid projective synchronization with time delay between system (8) and system (10).

In the following step, we would like to give the numerical simulations to verify the effectiveness of the previously designed controller. In the numerical simulations, the predictor-corrector algorithm [31] is utilized to solve the differential equations of the systems (8) and (10). The initial conditions are $\left(x_{1}(0), x_{2}(0), x_{3}(0)\right)=(1,1,1)$ and $\left(y_{1}(0), y_{2}(0), y_{3}(0)\right)=(0.5,0.5,0.5)$ and time delay is set as $\tau=0.2$. If the control gain matrix $\mathbf{K}$ is chosen as previously, the state synchronization variables and errors of system (8) and system (10) varying with time $t$ are shown in Figure 3. From those figures, it can be seen that the state variables of the system (8) and system (10) with time delay $\tau=0.2$ tend to evolve in the same direction, get closer with the evolution of time, and finally coincide under the desired scaling factors $\left(\lambda_{1}, \lambda_{2}, \lambda_{3}\right)=(1,-1,1)$; all the synchronization errors converge to zero. It implies that the hybrid projective synchronization with time delay between the fractional-order system (8) and system (10) is achieved.

3.2. Hybrid Projective Synchronization between the Hyperchaotic Fractional-Order Lorenz System and a New Hyperchaotic System. The fractional-order Lorenz system can be described as follows:

$$
\begin{aligned}
& D_{*}^{q} x_{1}=a\left(x_{2}-x_{1}\right)+x_{4}, \\
& D_{*}^{q} x_{2}=c x_{1}-x_{2}-x_{1} x_{3}, \\
& D_{*}^{q} x_{3}=x_{1} x_{2}-b x_{3}, \\
& D_{*}^{q} x_{4}=-x_{2} x_{3}+r x_{4},
\end{aligned}
$$

when $q=0.98$ and $(a, b, c, r)=(10,8 / 3,28,-1)$, the fractional-order Lorenz hyperchaotic attractor is shown in Figure 4.

A new modified four-dimensional hyperchaotic system with two nonlinearity terms which presented in [19] can be expressed as

$$
\begin{aligned}
& D_{*}^{q} y_{1}=e\left(y_{2}-y_{1}\right)+f y_{4}, \\
& D_{*}^{q} y_{2}=h y_{1}-g y_{1} y_{3}+\theta y_{4}, \\
& D_{*}^{q} y_{3}=-d y_{3}+y_{1} y_{2}, \\
& D_{*}^{q} y_{4}=-10 y_{2},
\end{aligned}
$$

when $q=0.98, e=10, f=15, h=40, \theta=10, d=$ 2.5 , the new four-dimensional fractional-order system (17) is hyperchaotic as depicted in Figure 5.

We consider system (16) as the drive system; the response system is described by the following differential equation:

$$
\begin{gathered}
D_{*}^{q} y_{1}=e\left(y_{2}-y_{1}\right)+f y_{4}+u_{1} \\
D_{*}^{q} y_{2}=h y_{1}-g y_{1} y_{3}+\theta y_{4}+u_{2} \\
D_{*}^{q} y_{3}=-d y_{3}+\beta y_{1} y_{2}+u_{3} \\
D_{*}^{q} y_{4}=-10 y_{2}+u_{4},
\end{gathered}
$$

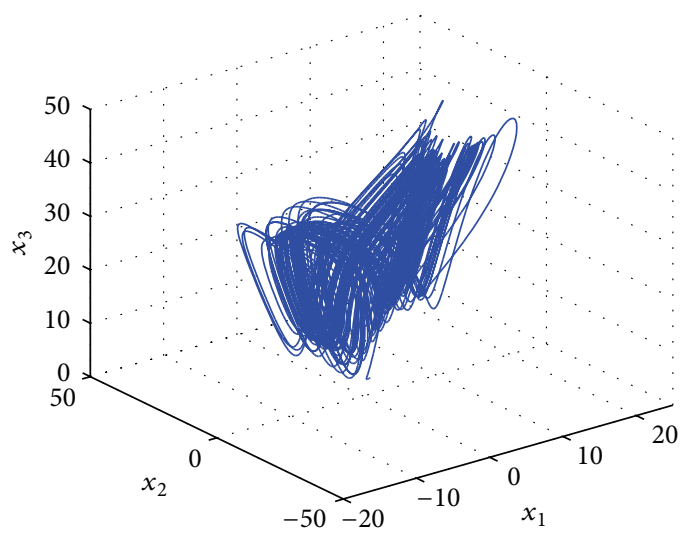

FIgURE 4: The attractor of the Lorenz system with $q=0.98$.

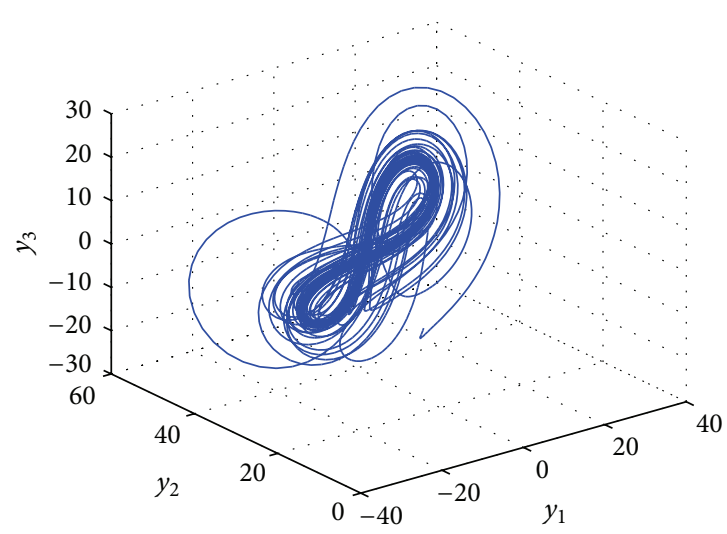

FIGURE 5: The attractor of the new system with $q=0.98$.

where $u_{i}(i=1,2,3,4)$ are the control functions to be determined in the later discussion.

For the sake of simplification, we can get

$$
\begin{aligned}
\mathbf{A}=\left[\begin{array}{cccc}
-a & a & 0 & 1 \\
c & -1 & 0 & 0 \\
0 & 0 & -b & 0 \\
0 & 0 & 0 & r
\end{array}\right], & \mathbf{F}(\mathbf{x})=\left(\begin{array}{c}
0 \\
-x_{1} x_{3} \\
x_{1} x_{2} \\
-x_{2} x_{3}
\end{array}\right), \\
\mathbf{B}=\left[\begin{array}{cccc}
-e & e & 0 & f \\
h & 0 & 0 & \theta \\
0 & 0 & -d & 0 \\
0 & -10 & 0 & 0
\end{array}\right], & \mathbf{G}(\mathbf{y})=\left(\begin{array}{c}
0 \\
-y_{1} y_{3} \\
y_{1} y_{2} \\
0
\end{array}\right) .
\end{aligned}
$$

Here, we choose scaling matrix as

$$
\Lambda=\left[\begin{array}{cccc}
1.5 & 1.5 & 0 & 0 \\
0 & -2 & 0 & 0 \\
0 & 0 & 3 & 0 \\
0 & 0 & 0 & -1
\end{array}\right]
$$




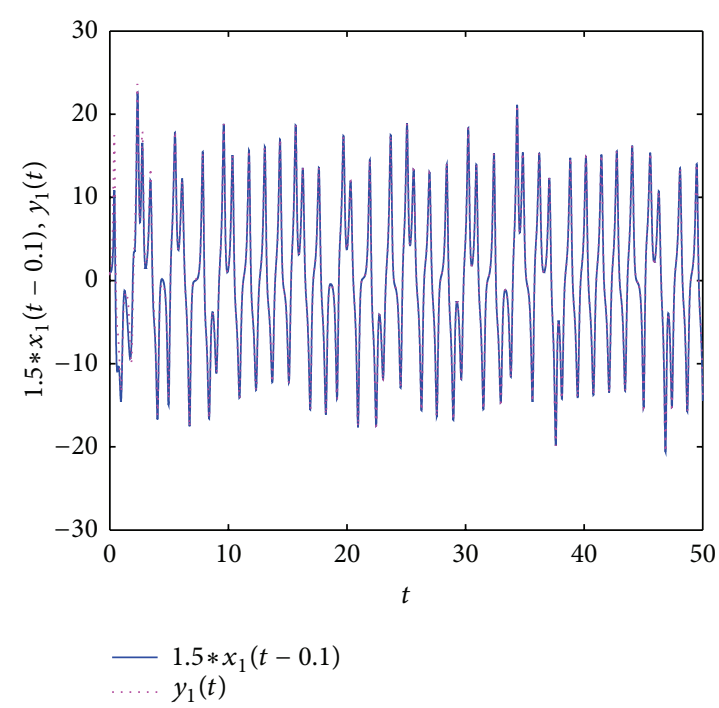

(a) State variables of $x_{1}$ and $y_{1}$

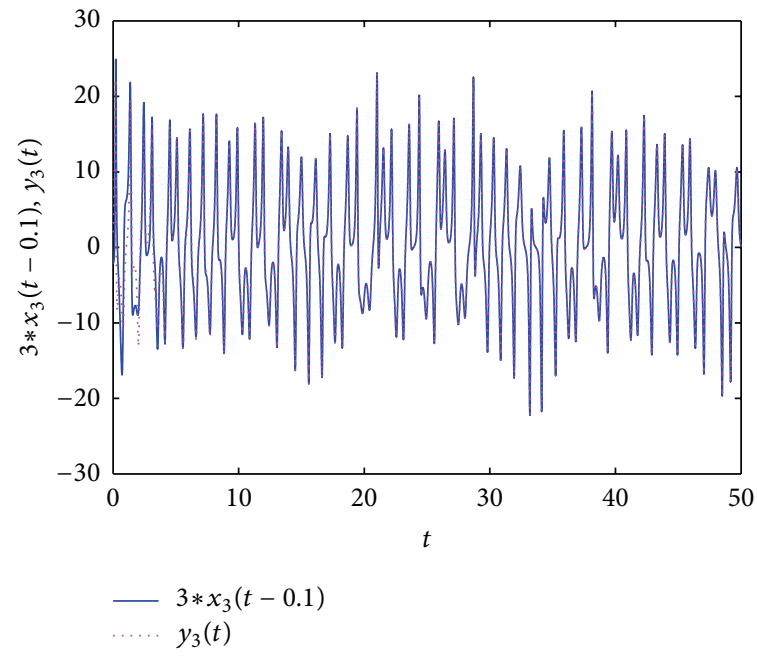

(c) State variables of $x_{3}$ and $y_{3}$

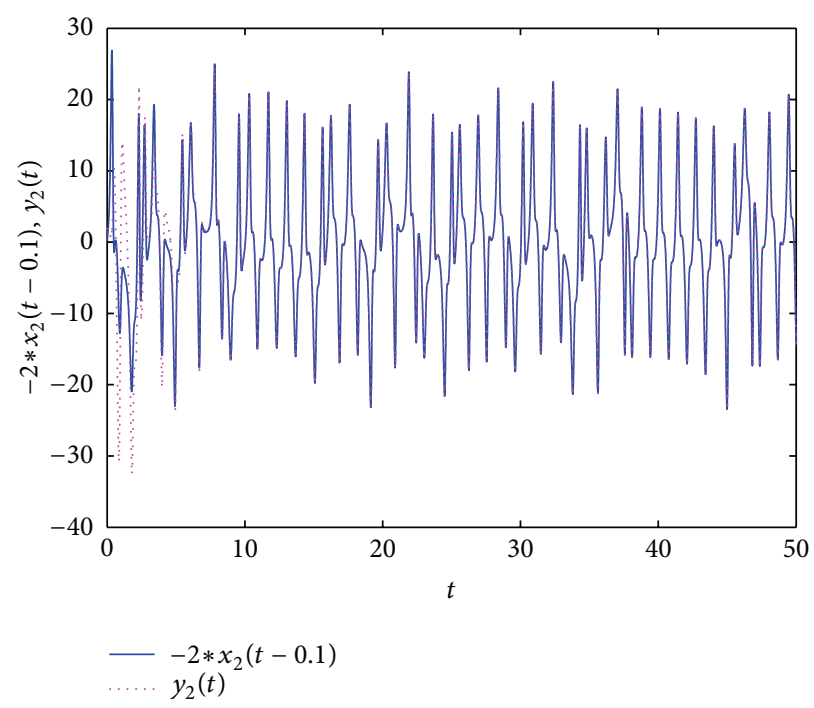

(b) State variables of $x_{2}$ and $y_{2}$

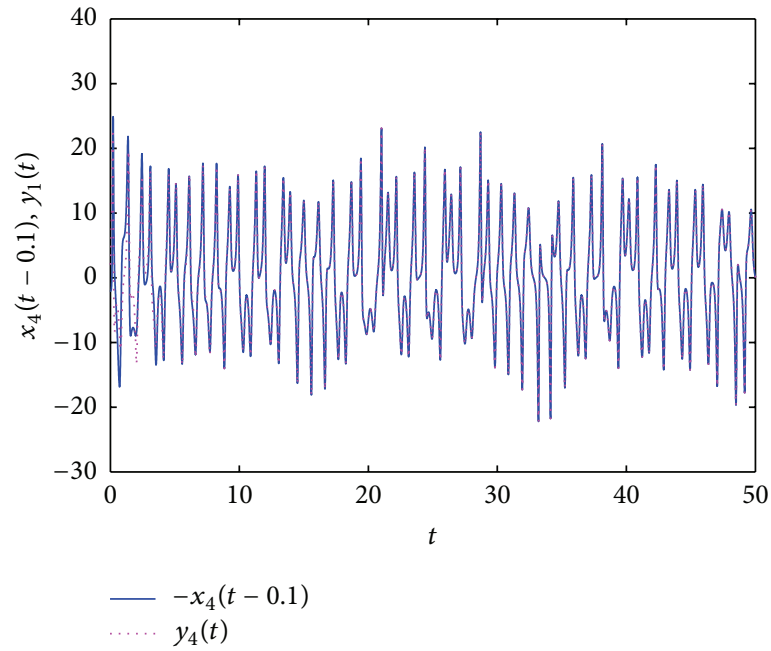

(d) State variables of $x_{4}$ and $y_{4}$

FIGURE 6: The state synchronization variables between the drive system (16) and response system (18) with time delay $\tau=0.1$ and scaling factors $\left(\lambda_{1}, \lambda_{2}, \lambda_{3}, \lambda_{4}\right)=(1.5,-2,3,-1)$.

The error system $\mathbf{e}(t)=\mathbf{y}(t)-\mathbf{\Lambda x}(t-\tau)$ can be obtain as

$$
\begin{gathered}
e_{1}=y_{1}-1.5\left(x_{1}(t-\tau)\right), \\
e_{2}=y_{2}+2\left(x_{2}(t-\tau)\right), \\
e_{3}=y_{3}-3\left(x_{3}(t-\tau)\right), \\
e_{4}=y_{4}+\left(x_{4}(t-\tau)\right) .
\end{gathered}
$$

From Theorem 6, we can obtain the controller $\mathbf{U}=\Lambda \mathbf{A x}(t-$ $\tau)+\Lambda \mathbf{F x}(t-\tau)-\mathbf{B} \mathbf{\Lambda} \mathbf{x}(t-\tau)+\mathbf{K e}(t), \mathbf{K} \in \mathbf{R}^{n \times n}$. Therefore, the error system is given in the following form:

$$
D_{*}^{q} \mathbf{e}=(\mathbf{B}+\mathbf{K}) \mathbf{e} .
$$

It follows from Theorem 6 that there exists a gain matrix $\mathbf{K}$ so that system (16) and system (18) realize the hybrid projective synchronization. For the sake of convenience, we can select all the eigenvalues $\lambda_{i}=-1$ of $\mathbf{B}+\mathbf{K}$ with the following matrix:

$$
\mathbf{K}=\left[\begin{array}{cccc}
9 & -10 & 0 & -15 \\
-40 & -1 & 0 & -10 \\
0 & 0 & -1.5 & 0 \\
0 & 10 & 0 & -1
\end{array}\right]
$$

which satisfy $\left|\arg \left(\lambda_{i}\right)\right|>0.98 \pi / 2, i=1,2,3, \ldots$. In other words, we can realize the hybrid projective synchronization between system (16) and system (18).

Analogously, we also would like to give the numerical simulations to verify the effectiveness of the previously designed controller. The initial values are chosen as $\left(x_{1}(0), x_{2}(0), x_{3}(0), x_{4}(0)\right)=(0.1,2,3,0.5)\left(y_{1}(0), y_{2}(0)\right.$, $\left.y_{3}(0), y_{4}(0)\right)=(0.5,1,1,0.5)$. Time delay is taken as $\tau=0.1$. The control gain matrix $\mathbf{K}$ is selected as mentioned above (23). Figure 6 shows that the state synchronization variables 


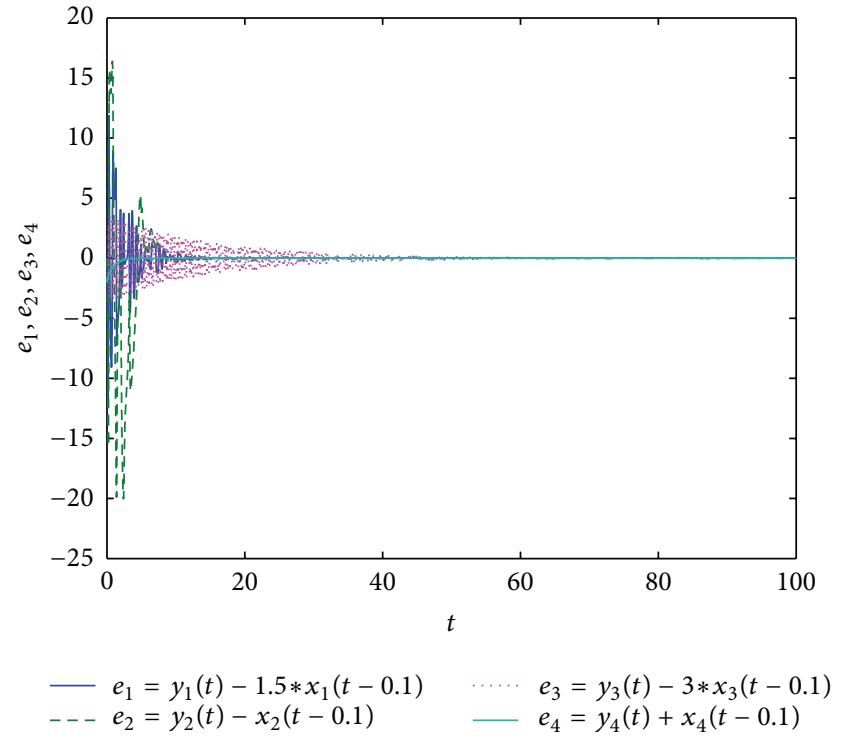

FIGURE 7: The time evolution of synchronization errors with $q=$ 0.85 .

of system (16) and system (18) vary with time. From Figure 6, it can be seen that the state variables of system (16) and system (18) with time delay $\tau=0.1$ tend to evolve in the same direction, get closer with the evolution of time, and finally coincide under the desired scaling factors $\left(\lambda_{1}, \lambda_{2}, \lambda_{3}, \lambda_{4}\right)=$ $(1.5,-2,3,-1)$, where the red line stands for the response system, and blue line denotes the drive system. Which means that the hybrid projective synchronization with time delay between the fractional-order system (16) and system (18) is achieved.

In order to discuss the influence of the fractional order on the synchronization process, we again use the previous hyperchaotic fractional-order system of (16) and (18) to present another example. We fix the parameters as stated in (16) and (18) and test the proposed synchronization scheme for other values of order when $q=0.85, q=0.98$, and $q=1$, respectively. Figures $7-9$ depict the time evolution of the synchronization errors with different order; in this simulation, we set $\tau=0.1$; it can be noted that the smaller the fractional order is, the worse the synchronization performance turns. For instance, when the fractional order $q=0.85$, the synchronization is achieved at $t=40 \mathrm{~s}$; the result is shown in Figure 7. However, when the fractional order $q=0.98$, the synchronization can be realized before $t=20 \mathrm{~s}$ as shown in Figure 8. In particular, when $q=1$, the fractional order dynamical chaotic system is simplified to the integer order dynamical chaotic system; Figure 9 shows that the synchronization time is about $t=5 \mathrm{~s}$. It is obvious that the synchronization rate of the integer order chaotic system is much faster than that of the fractional-order one, and the synchronization effect is much better than that of the fractional-order one.

\section{Conclusions}

In this paper, the hybrid projective synchronization with time delay of chaotic systems has been investigated. Based on

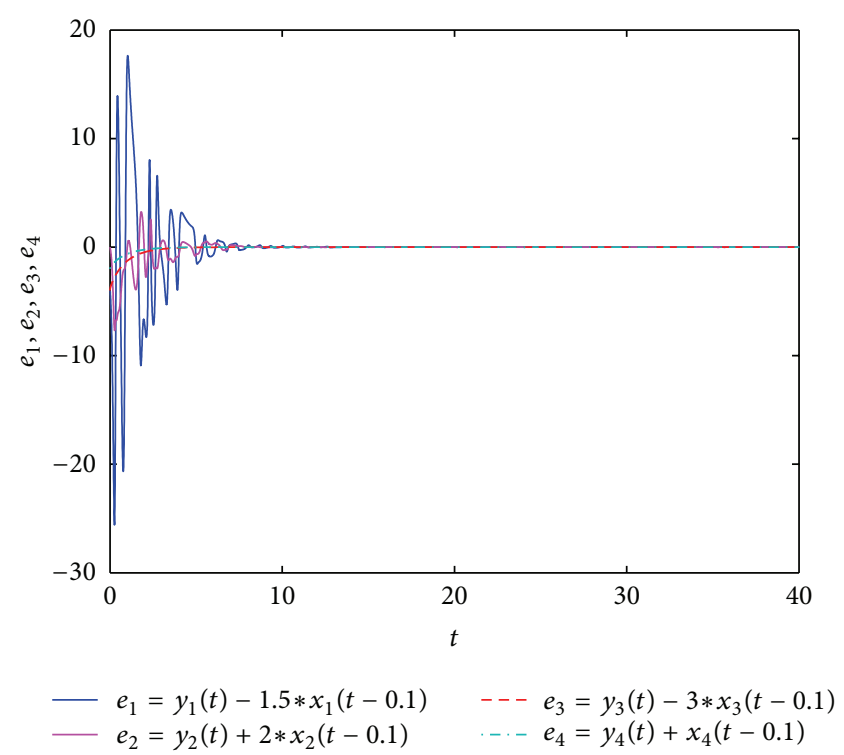

FIgURE 8: The time evolution of synchronization errors with $q=$ 0.98 .

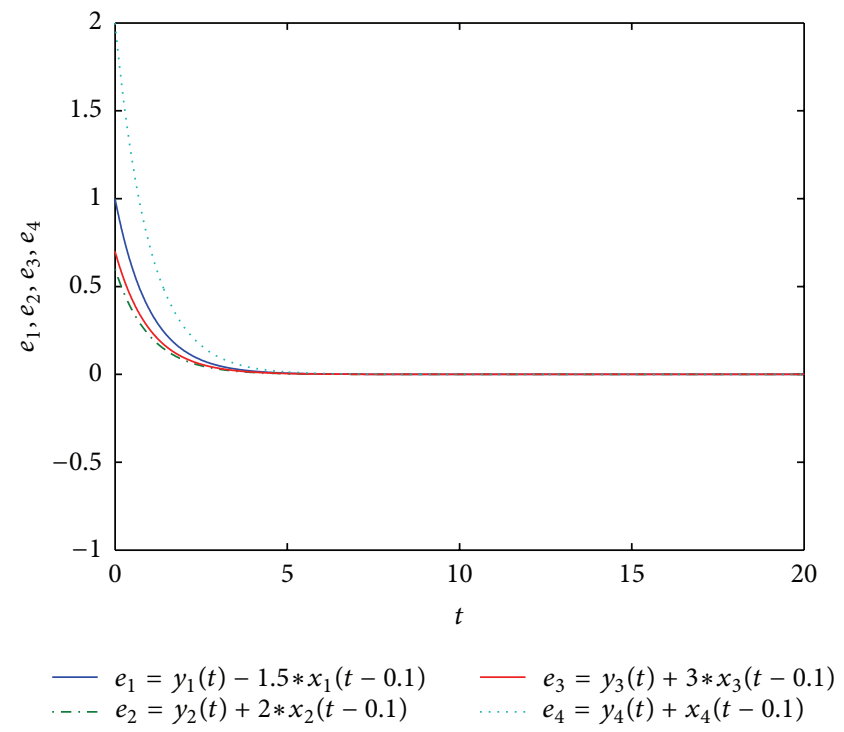

FIGURE 9: The time evolution of synchronization errors with $q=1$.

the stability theory of fractional-order differential equations, an appropriate controller is designed to realize the hybrid projective synchronization of chaotic and hyperchaotic systems. Some numerical simulations are presented to show the applicability and feasibility of the proposed approach.

In addition, as the fractional order imposes direct effect on the chaotic behavior of the nonlinear dynamical system, it may affect the synchronization performance of the fractional order systems. We use the above fractional-order chaotic systems to present detailed discussion. Numerical evidence shows that the bigger the fractional order is, the better the synchronization performance turns. Meanwhile, the synchronization rate of the integer-order chaotic system is 
much faster than that of the fractional-order one, and the synchronization effect is much better than that of the fractionalorder one. It can be seen that the order is an important intrinsic parameter on the synchronization process.

\section{Acknowledgments}

The authors thank the reviewers for their careful reading and providing some pertinent suggestions. This work is supported by the National Natural Science Foundation of China (NSFC) under the Grant no. 11172223.

\section{References}

[1] G. W. Leibniz, Mathematics Schiften, Georg Olms Verlagsbuchhandlung, Hildesheim, Germany, 1962.

[2] A. C.-L. Chian, F. A. Borotto, E. L. Rempel, and C. Rogers, "Attractor merging crisis in chaotic business cycles," Chaos, Solitons and Fractals, vol. 24, no. 3, pp. 869-875, 2005.

[3] A. C.-L. Chian, E. L. Rempel, and C. Rogers, "Complex economic dynamics: chaotic saddle, crisis and intermittency," Chaos, Solitons and Fractals, vol. 29, no. 5, pp. 1194-1218, 2006.

[4] K. Sasakura, "On the dynamic behavior of Schinasi's business cycle model," Journal of Macroeconomics, vol. 16, no. 3, pp. 423444, 1994.

[5] H. H. Lee and C.-S. Tsai, "Analytical model of viscoelastic dampers for seismic mitigation of structures," Computers and Structures, vol. 50, no. 1, pp. 111-121, 1994.

[6] J. Bisquert, G. Garcia-Belmonte, and F. Fabregat-Santiago, "The role of instrumentation in the process of modeling real capacitors," IEEE Transactions on Education, vol. 43, no. 4, pp. 439-442, 2000.

[7] B. M. Vinagre and V. Feliu, "Modeling and control of dynamic systems using fractional calculus: application to electrochemical processes and flexible structures," in Proceedings of the 41st IEEE Conference Decision Control, Las Vegas, Nev, USA, 2002.

[8] V. Daftardar-Gejji and S. Bhalekar, "Chaos in fractional ordered Liu system," Computers \& Mathematics with Applications, vol. 59, no. 3, pp. 1117-1127, 2010.

[9] S. Bowong, "Adaptive synchronization between two different chaotic dynamical systems," Communications in Nonlinear Science and Numerical Simulation, vol. 12, no. 6, pp. 976-985, 2007.

[10] I. Petras, "A note on the fractional-order Chua's system," Chaos, Solitons and Fractals, vol. 38, no. 2, pp. 140-144, 2008.

[11] S. H. Hosseinnia, R. Ghaderi, and A. Ranjbar, "Synchronization of gyro systems via fractional-order adaptive controller," in New Trends in Nanotechnology and Fractional Calculus Applications, Springer, New York, NY, USA, 2010.

[12] I. Osorio and Y.-C. Lai, "A phase-synchronization and randommatrix based approach to multichannel time-series analysis with application to epilepsy," Chaos, vol. 21, no. 3, Article ID 033108, 2011.

[13] J. Lu, "Generalized (complete, lag, anticipated) synchronization of discrete-time chaotic systems," Communications in Nonlinear Science and Numerical Simulation, vol. 13, no. 9, pp. 1851-1859, 2008.

[14] X. Gao and J. Yu, "Chaos in the fractional order periodically forced complex Duffing's oscillators," Chaos, Solitons and Fractals, vol. 24, no. 4, pp. 1097-1104, 2005.
[15] W. H. Deng and C. P. Li, "Chaos synchronization of the fractional Lü system,” Physica A, vol. 353, no. 1-4, pp. 61-72, 2005.

[16] C. Li and G. Peng, "Chaos in Chen's system with a fractional order," Chaos, Solitons \& Fractals, vol. 22, no. 2, pp. 443-450, 2004.

[17] J. G. Lu and G. Chen, "A note on the fractional-order Chen system," Chaos, Solitons and Fractals, vol. 27, no. 3, pp. 685-688, 2006.

[18] J. Huang, "Chaos synchronization between two novel different hyperchaotic systems with unknown parameters," Nonlinear Analysis: Theory, Methods \& Applications, vol. 69, no. 11, pp. 4174-4181, 2008.

[19] L. Liu, D. Liang, and C. Liu, "Nonlinear state-observer control for projective synchronization of a fractional-order hyperchaotic system," Nonlinear Dynamics, vol. 69, no. 4, pp. 19291939, 2012.

[20] J. Cao, D. W. C. Ho, and Y. Yang, "Projective synchronization of a class of delayed chaotic systems via impulsive control," Physics Letters A, vol. 373, no. 35, pp. 3128-3133, 2009.

[21] W. He, F. Qian, J. Cao, and Q.-L. Han, "Impulsive synchronization of two nonidentical chaotic systems with time-varying delay," Physics Letters A, vol. 375, no. 3, pp. 498-504, 2011.

[22] W. He and J. Cao, "Generalized synchronization of chaotic systems: an auxiliary system approach via matrix measure," Chaos, vol. 19, no. 1, Article ID 013118, 2009.

[23] G.-H. Li, "Modified projective synchronization of chaotic system," Chaos, Solitons and Fractals, vol. 32, no. 5, pp. 1786-1790, 2007.

[24] G. H. Erjaee and M. Alnasr, "Phase synchronization in coupled Sprott chaotic systems presented by fractional differential equations," Discrete Dynamics in Nature and Society, vol. 2009, Article ID 753746, 10 pages, 2009.

[25] J.-F. Zhang, Q.-Y. Pei, and X.-L. Zhang, "A new kind of nonlinear phenomenon in coupled fractional-order chaotic systems: coexistence of anti-phase and complete synchronization," Chinese Physics B, vol. 20, no. 8, Article ID 080503, 2011.

[26] S. Wang, Y. Yu, and M. Diao, "Hybrid projective synchronization of chaotic fractional order systems with different dimensions," Physica A, vol. 389, no. 21, pp. 4981-4988, 2010.

[27] Y. Chen, X. Chen, and S. Gu, "Lag synchronization of structurally nonequivalent chaotic systems with time delays," Nonlinear Analysis: Theory, Methods \& Applications, vol. 66, no. 9, pp. 1929-1937, 2007.

[28] T.-C. Lin and T.-Y. Lee, "Chaos synchronization of uncertain fractional-order chaotic systems with time delay based on adaptive fuzzy sliding mode control," IEEE Transactions on Fuzzy Systems, vol. 19, no. 4, pp. 623-635, 2011.

[29] D. Matignon, "Stability results for fractional differential equations with applications to control processing," in Proceeeding of the IEEE-SMC Computational Engineering in Systems Applications Multi-Conference (IMACS '96), vol. 2, pp. 963-968, Lille, France, 1996.

[30] L. P. Chen, Y. Chai, and R. C. Wu, "Lag projective synchronization in fractional-order chaotic (hyper-chaotic) systems," Physics Letters A, vol. 375, no. 35, pp. 2099-2110, 2011.

[31] K. Diethelm, N. J. Ford, and A. D. Freed, "A predictor-corrector approach for the numerical solution of fractional differential equations," Nonlinear Dynamics, vol. 29, no. 1-4, pp. 3-22, 2002. 


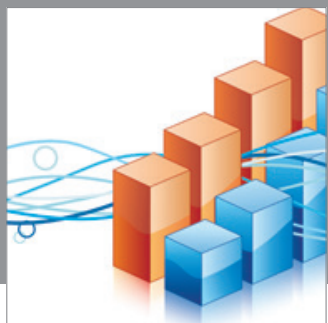

Advances in

Operations Research

mansans

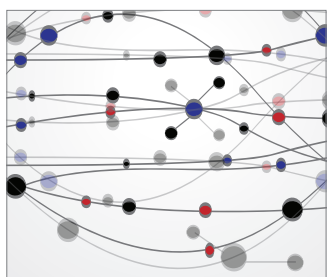

The Scientific World Journal
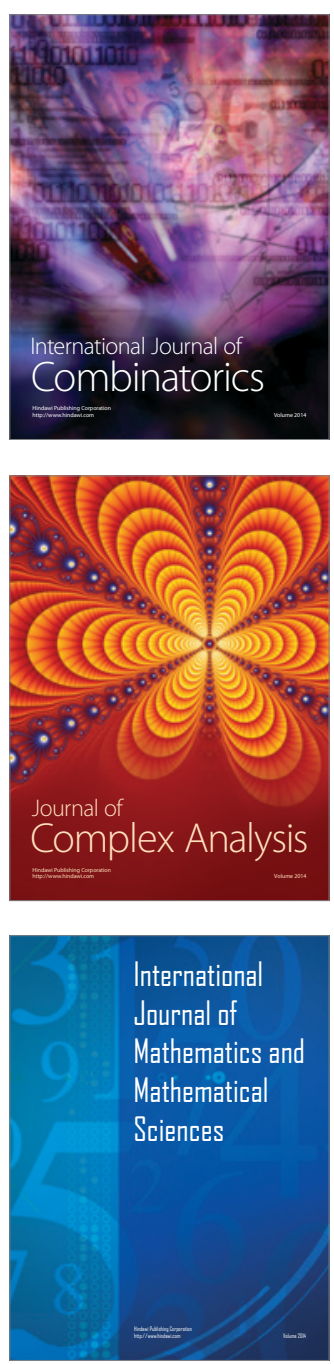
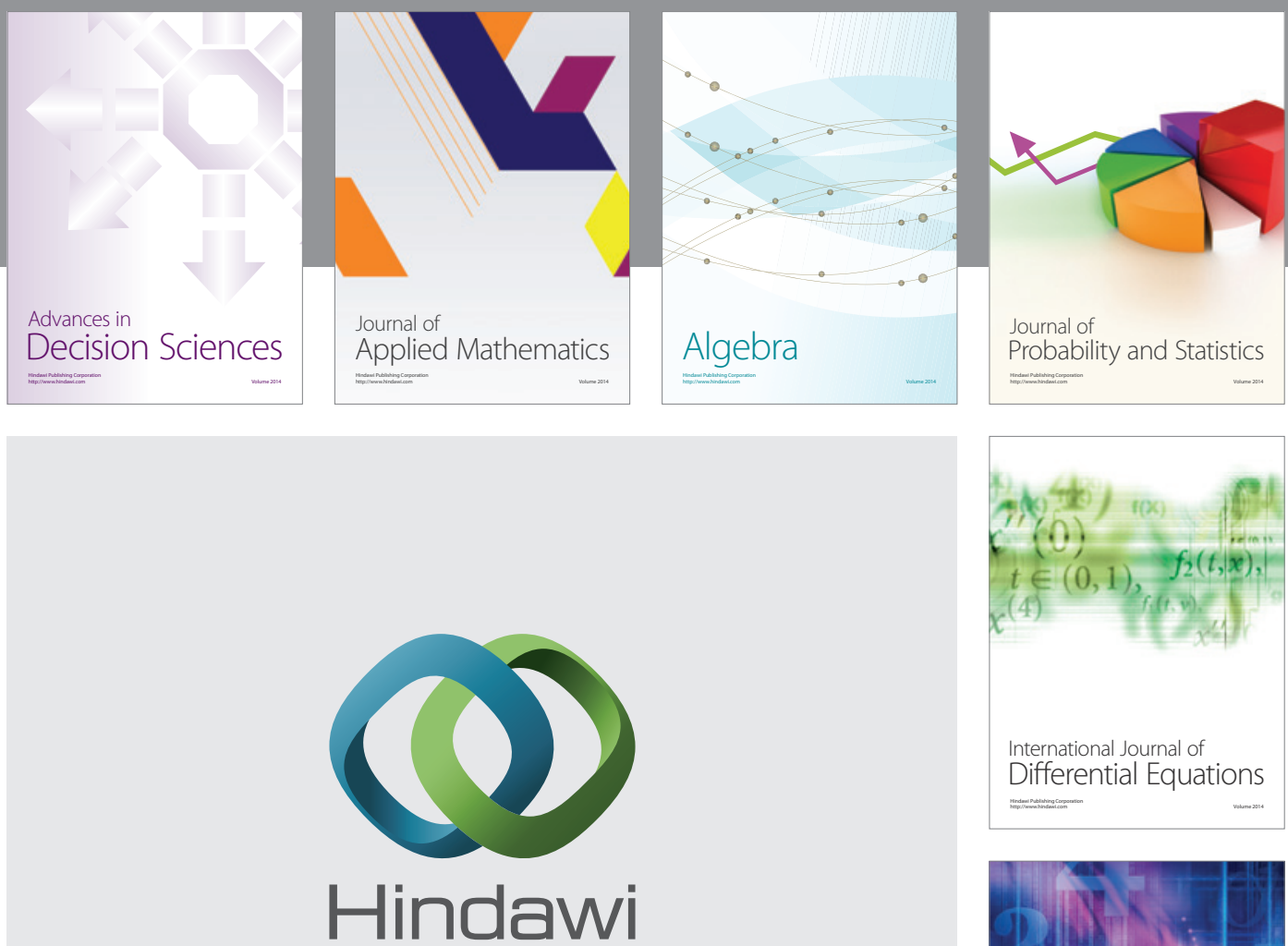

Submit your manuscripts at http://www.hindawi.com
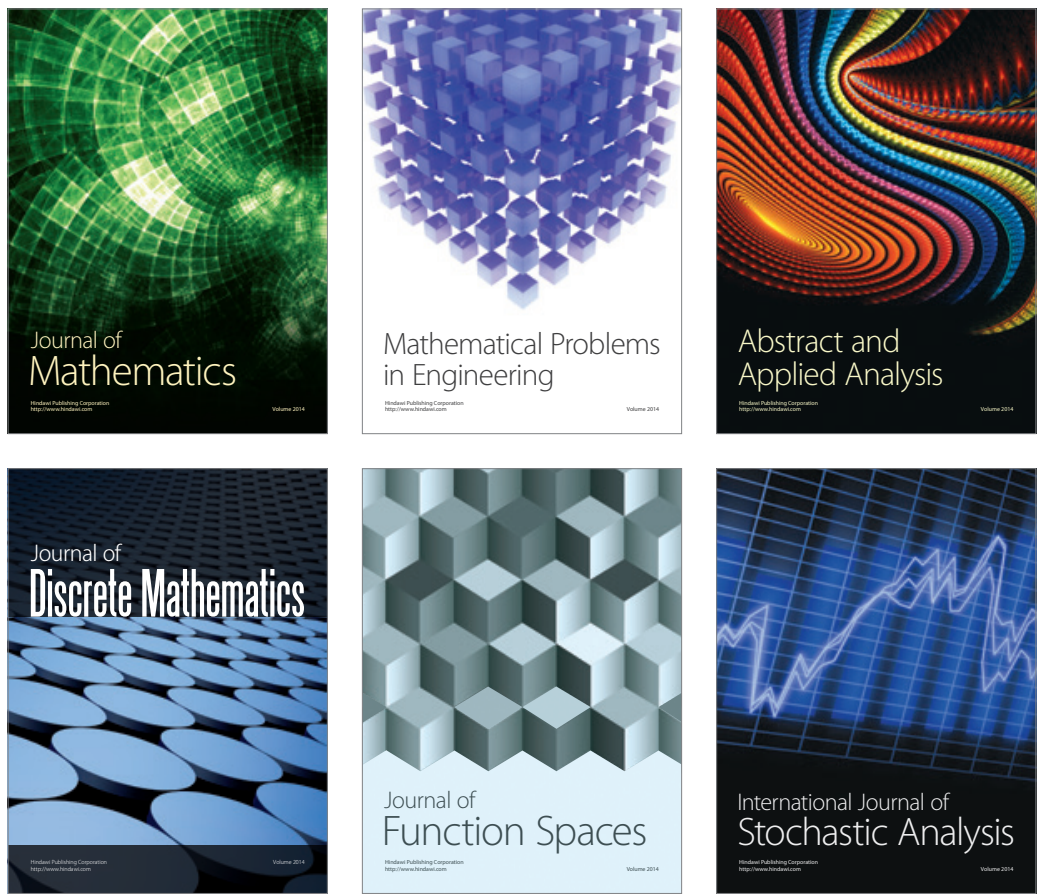

Journal of

Function Spaces

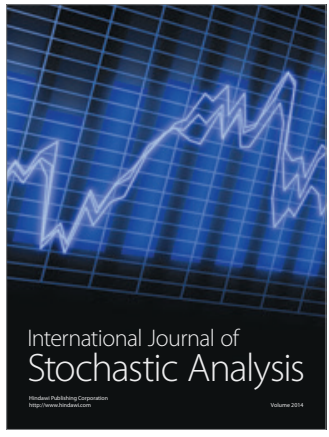

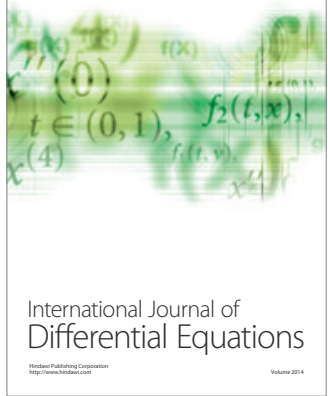
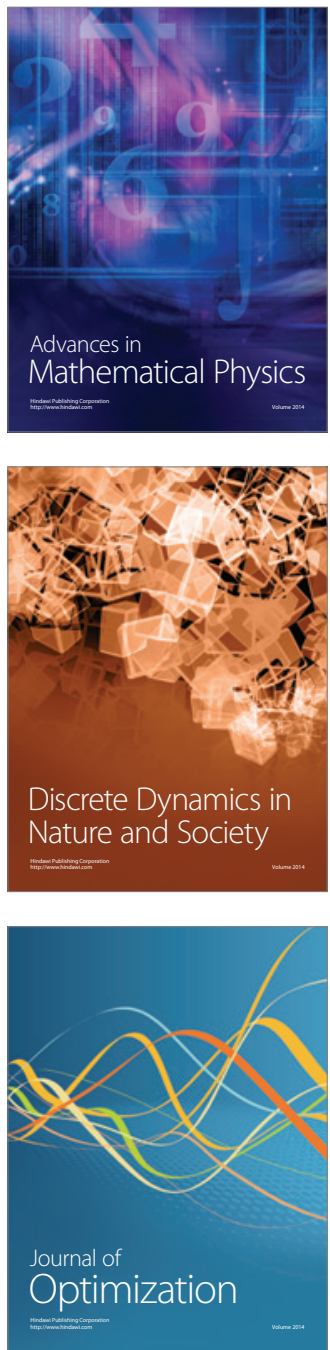